\title{
Quelle recherche dans un ministère? Témoignage d'une chargée de mission, Marie-Christine Bagnati ${ }^{\star}$ Propos recueillis par Marcel Jollivet
}

\author{
Marie-Christine Bagnati ${ }^{1}$ et Marcel Jollivet ${ }^{2, *}$ \\ ${ }^{1}$ Chargée de mission Gouvernance de l'environnement, du développement durable et de la recherche, CGDD, \\ Ministère de la Transition écologique et solidaire, France \\ ${ }^{2}$ Sociologie, association NSS-Dialogues, Ladyss, CNRS, Université Paris Nanterre la Défense, Nanterre, France
}

Marie-Christine Bagnati a été chargée de mission au service de la recherche du ministère en charge de l'environnement pendant sept ans $(2010-2017)^{1}$. Elle fait part de l'expérience qu'elle a vécue en vue d'inciter à une réflexion collective sur la recherche finalisée en appui aux politiques publiques.

NSS : Pouvez-vous nous éclairer sur la structure du ministère dont vous dépendez? Quelle place occupe-t-elle dans son organigramme général ?

Marie-Christine Bagnati : Mon poste est rattaché au service de la recherche qui est l'une des trois entités de la direction de la recherche et de l'innovation. Cette direction a été placée au sein du Commissariat général au développement durable dans le grand ministère créé en 2007 par fusion du ministère de l'Environnement et de celui de l'Équipement.

NSS : Quel est le titre exact du poste que vous y occupez? Depuis quand l'occupez-vous ?

Marie-Christine Bagnati: J'ai été nommée dans mon poste en qualité de chargée de mission. Ma nomination a pris effet en 2010 .

\footnotetext{
‡ Voir dans ce numéro le texte d'introduction de Marcel Jollivet.

* Auteur correspondant :

marcel.jollivet@parisnanterre.fr

${ }^{1}$ M.-C. Bagnati était également chef de projet pour le pilotage de la réflexion transversale sur la recherche finalisée en appui aux politiques publiques du ministère. À ce jour, elle n'exerce plus ces fonctions.
}

\section{NSS : Quelles sont vos fonctions dans ce poste?}

Marie-Christine Bagnati: Il faut distinguer mes fonctions initiales et celles que j'ai eues ensuite. J'ai eu au départ les fonctions communes à la plupart des chargés de mission du service de la recherche, notamment le suivi d'au moins un programme de recherche. Lorsque j'ai été nommée, le ministère disposait encore de crédits incitatifs lui permettant de conduire sa propre programmation scientifique. J'ai ainsi pris la responsabilité de deux programmes. L'un d'eux, le programme "Concertation décision environnement», correspondait au format et aux règles de la plupart des programmes de recherche du ministère. Mais l'autre, le programme REPERE (Réseau d'échange et de projets sur le pilotage de la recherche et de l'expertise), celui qui a été créé plus tardivement, peu avant mon arrivée, présentait un caractère très expérimental puisqu'il s'agissait de concevoir et d'animer une réflexion sur la recherche participative en vue d'identifier les voies de son développement, en donnant une large place aux associations environnementales.

NSS : Vous parlez de fonctions nouvelles que vous auriez eues ensuite. De quoi s'agit-il ?

Marie-Christine Bagnati: Deux ans après ma prise de fonction, le périmètre du poste a été élargi. Claire Hubert (alors chef du service de la recherche) m'a demandé d'élaborer un projet présentant un intérêt pour la direction de la recherche et de l'innovation. Or, depuis mon arrivée en 2010, en jouant mon rôle de chargée de mission auprès du programme de recherche "Concertation décision environnement», j'avais été amenée à en observer attentivement le fonctionnement. J'avais ainsi constaté la diversité et la 
complémentarité des approches scientifiques et disciplinaires mises en œuvre, des problématiques abordées et des terrains d'étude des équipes de recherches. J'ai pu observer également le processus de montée en généralité des résultats d'une vingtaine de projets jusqu'à l'élaboration de recommandations de portée générale en réponse aux questionnements qui avaient fondé la reprise du programme en 2008. Ce travail remarquable m'a convaincu que les programmes du ministère constituaient un modèle particulièrement pertinent pour à la fois faire progresser la connaissance et poursuivre une finalité d'action publique. Cette expérience m'a confortée dans l'idée de conduire une étude transversale aux programmes de recherche du ministère pour rendre visibles les spécificités de la recherche qui était ainsi financée et ses apports pour les politiques publiques. C'est ce que j'ai proposé. C'est ainsi que j'ai été amenée à prendre en charge, pour le compte du service de la recherche, la thématique de la recherche finalisée en appui aux politiques publiques.

Par ailleurs, au même moment, il fallait adapter les missions du service de la recherche à la situation nouvelle dans laquelle il se trouvait, puisque la disparition des crédits incitatifs ne lui permettait plus de lancer de nouveaux appels à projets, et encore moins de nouveaux programmes pour mobiliser les chercheurs sur des problématiques émergentes en lien avec les politiques publiques du ministère. Comme j'avais également en charge le programme REPERE, j'étais sensible aux avancées qu'il avait déjà permises dans le domaine des recherches participatives et convaincue que ces recherches étaient susceptibles de renouveler la façon de concevoir l'élaboration et la mise en œuvre des politiques publiques du ministère. C'est la raison pour laquelle j'ai proposé de mettre en place, dans le cadre d'une prolongation du programme REPERE, une "Commission pluraliste». Composée de chercheurs, de représentants d'organismes et d'agences de recherche, d'associations environnementales, de fondations et de collectivités territoriales, cette commission constitue un nouveau cadre pour poursuivre autrement, c'est-à-dire sans crédits incitatifs, et collectivement, cette réflexion sur la recherche participative. Installée en juin 2015, elle offre un espace de dialogue et d'expérimentation pour traiter des enjeux du développement de cette démarche de recherche, en faire reconnaître les apports, mettre en débat ses pratiques afin d'améliorer la qualité scientifique des connaissances ainsi produites; et faire reconnaître sa portée démocratique. La commission a ainsi conduit une réflexion approfondie sur les critères et modalités de cette recherche qui est une question centrale pour sa prise en compte dans la programmation de la recherche. Dans cette perspective, elle a lancé un premier appel à manifestation d'intérêt, qui a reçu un accueil révélateur d'une attente. Par la diversité des acteurs impliqués et la diffusion des travaux à différentes étapes de la réflexion, REPERE a amplifié la visibilité de la recherche participative, favorisé l'émergence de nouvelles initiatives et la montée en compétences de celles et ceux qui les portent.

NSS : Lorsque vous avez pris en main le dossier des programmes de recherche pilotés par le ministère, quelle était la situation?

Marie-Christine Bagnati : À mon arrivée, en 2010, le ministère disposait d'une vingtaine de programmes de recherche et avait encore une capacité d'engagement de $18 \mathrm{M} €$ annuels pour soutenir, dans ce cadre, des travaux de recherche. La diminution des crédits incitatifs s'est amplifiée jusqu'à leur disparition en 2015. Il n'en était que plus important d'essayer de sauvegarder ce qui pouvait l'être d'une expérience de programmation scientifique d'origine ministérielle aussi longue (de l'ordre de la quarantaine d'années), aussi massive, aussi variée et originale dans sa démarche. D'où ma proposition d'étude transversale, dont j'ai assuré le suivi en commanditant les deux expertises de l'association Natures Sciences Sociétés-Dialogues et d'AScA, et en faisant en sorte que leurs résultats aient le maximum d'audience possible, via notamment le colloque de juillet $2016^{2}$ et cette publication qui en est issue.

NSS : Pourquoi avez-vous choisi cette fonction? Comment avez-vous pris en main vos responsabilités? Étiez-vous préparée?

Marie-Christine Bagnati: J'avais précédemment exercé différentes fonctions au ministère de la Recherche dont certaines m'ont amenée à réfléchir au fonctionnement du système de la recherche et de l'innovation et à l'articulation entre orientation et programmation de la recherche. J'ai notamment participé à une étude pilotée par Rémi Barré qui portait sur la caractérisation des processus de programmation de la recherche, en particulier sur ceux des organismes de recherches et des ministères. J'ai par la suite accompagné sur le plan méthodologique le dispositif de réflexion, piloté par Jean-François Stéphan alors directeur scientifique du département Environnement, Planète-Univers, Espace au ministère de la Recherche, pour élaborer une stratégie « enseignement supérieur, recherche et innovation » pour et avec les départements et territoires ultramarins. Cette expérience intense m'a permis de développer une meilleure connaissance des enjeux territoriaux de la

\footnotetext{
${ }^{2}$ Colloque «Transition écologique : pour une interaction entre politiques publiques et recherches finalisées » qui s'est tenu les 5 et 6 juillet 2016 au ministère de l'Environnement, de l'Énergie et de la Mer à La Défense (http://www2.developpe ment-durable.gouv.fr/Transition-ecologique-pour-une.html).
} 
recherche. Il y a une cohérence et une forme de continuité - à laquelle je tenais beaucoup - entre ces expériences et les fonctions exercées au sein du service de la recherche, en particulier parce qu'elles permettent d'appréhender ensemble les enjeux scientifiques et sociétaux de la recherche et pour ces derniers leur implication en termes d'action publique.

NSS : Qu'est-ce qui a le plus compté pour vous dans les tâches qui vous incombaient? Quelles initiatives avez-vous prises? Lesquelles vous ont donné le plus de satisfaction?

Marie-Christine Bagnati: Les travaux portant sur les formes de la recherche sont sans doute ceux qui m'ont donné le plus de satisfaction. Ces deux sujets m'ont permis d'interagir à différents niveaux avec une très grande diversité d'acteurs dont j'ai pu apprécier le professionnalisme, l'engagement et l'envie de travailler ensemble. Parler d'une expérience professionnelle et humaine exceptionnelle est sans doute une expression un peu convenue. Mais elle s'applique particulièrement dans ce cas.

NSS : Comment votre fonction s'articule-t-elle avec celles de vos collègues au sein du service et celle des chercheurs?

Marie-Christine Bagnati: Portant sur l'ensemble des programmes, l'étude transversale sur la recherche finalisée en appui aux politiques publiques que j'ai mise en œuvre m'a amenée à interagir avec l'ensemble des chargés de mission du service qui ont participé aux enquêtes, ainsi qu'avec les présidents des conseils scientifiques des programmes, eux-mêmes chercheurs, qui ont contribué à cette réflexion. Avec les chercheurs en général, il s'agit de contribuer au mieux à l'essaimage de leurs travaux et de créer les conditions de leur contribution aux politiques publiques. Faute de crédits incitatifs, cela peut passer par des dispositifs d'animation comme celui mis en place pour REPERE. Mais les crédits incitatifs restent évidemment déterminants.

NSS : Quelles sont les difficultés majeures auxquelles vous vous êtes heurtée?

Marie-Christine Bagnati : Une difficulté pour moi a été de prendre en marche le programme «Concertation décision environnement » alors qu'il arrivait en phase de capitalisation. Une communauté s'était solidement constituée au fil du temps et il n'était pas simple de s'y incorporer et d'y prendre ses marques. Cela confirme que le programme avait pris consistance. Je me suis attachée à obtenir les meilleures conditions possibles pour valoriser les résultats de ce programme. Une autre difficulté est très certainement liée aux questions abordées. Traitant des rapports entre sciences et société, elles divisent les communautés scientifiques. Les concepts de recherche finalisée en appui aux politiques publiques et de recherche participative soulèvent des objections tant sur le fond, c'est-à-dire du point de vue scientifique, que parce qu'ils sont ressentis, à tort me semble-t-il, comme remettant en cause l'autonomie des chercheurs. Il me semble surtout qu'ils interpellent la responsabilité $\mathrm{du}$ chercheur face aux grands défis sociétaux et environnementaux.

Citation de l'article : Bagnati M.-C., Jollivet M., 2017. Quelle recherche dans un ministère ? Témoignage d'une chargée de mission, Marie-Christine Bagnati. Nat. Sci. Soc. 25, S18-S20. 